\title{
Development of an Information- interoperable Environment Based on Open Technologies for Lean Production Systems
}

\author{
Toshiaki Kimura $^{1}$, Hirohisa Tezuka ${ }^{2}$ and Yuichi Kanda ${ }^{3}$ \\ ${ }^{1}$ Japan Society for the Promotion of Machine Industry, \\ 1-1-12, Hachiman-cho, Higashikurume-city, Tokyo, 203-0042 Japan \\ WWW homepage: http://www.tri.jspmi.or.jp/english/index.html \\ ${ }^{2}$ Nippon Telegraph and Telephone Corp., \\ 3-9-11, Midori-cho, Musashino-city, Tokyo, 180-8585 Japan \\ WWW homepage: http://www.ntt.co.jp/index_e.html \\ 3 Toyo University, \\ 2100 Kujirai, Kawagoe-city, Saitama, 350-8585 Japan \\ WWW homepage: http://www.eng.toyo.ac.jp/eng/index.html
}

\begin{abstract}
Lean production systems require an information-interoperable environment that can process diverse factory information for all workers to do KAIZEN activities. Moreover, the information-interoperable environment should be connected to an inter-enterprise collaboration environment. For those reasons, an Information-Interoperable Environment (IIE) that can connect device-level information and Manufacturing Execution System (MES)-level information was developed using Open Robot (or Resource) interface with the Network (ORiN). Moreover, CaoSQL2FDML for changing the IIE's information to Factory Data Markup Language (FDML) information was developed for inter-enterprise collaboration. This paper describes development of IIE including CaoSQL2FDML, prototype system development, and results of the system's operational testing.
\end{abstract}

\section{Introduction}

Rapid and flexible production is sought through the use of informationinteroperable production functions such as supply chain, manufacturing planning, manufacturing execution, and device controls for adapting to increasingly diverse product demands and shortening product life-cycles. A production culture is necessary to carry out various tasks through cooperation among workers for lean production. Moreover, an environment in which all workers can do KAIZEN activities using diverse information from factories is important.

Please use the following format when citing this chapter:

Kimura, T., Tezuka, H. and Kanda, Y., 2008, in IFIP International Federation for Information Processing, Volume 257, Lean Business Systems and Beyond, Tomasz Koch, ed.; (Boston: Springer), pp. 121-128. 
However, many de facto standards for information systems depend on the device industry, even though multi-devices such as machine tools and robots exist in factories. Therefore, information acquisition at factories from the device-level for KAIZEN activities is difficult. Development of an information-interoperable environment for factories that considers the relation between the device-level and the MES-level is increasingly important. In addition, a mechanism to connect the information-interoperable environment and inter-enterprise collaboration systems is required.

For that purpose, Open Robot (or Resource) interface with the Network (ORiN) [1] was developed as an open technology by the ORiN Forum for robots. Today, however, development of gateway systems between ORiN and other standards such as OPC [2], CC-Link [3], Profibus [4], and DeviceNet [5] are active in the ORiN Forum. Therefore, an Information-Interoperable Environment (IIE) [6] that can connect device-level information and MES-level information has been developed using ORiN. Moreover, CaoSQL2FDML, which is useful for changing the IIE's information to Factory Data Markup Language (FDML) [7] information, has been developed for inter-enterprise collaboration. The FDML is also an open resource that is useful for Application Service Provider (ASP) services. The present report describes developments of IIE, CaoSQL2FDML, a prototype system based on these technologies, and system evaluation.

\section{Development of IIE}

\subsection{Requirements of information-interoperable environment}

An information-interoperable environment that can handle diverse information from factories for all workers to do KAIZEN activities is necessary for lean production systems. Figure 1 presents requirements of the information-interoperable environment. The bottom of Fig. 1, Manufacturing systems, shows that the reference architecture of a manufacturing system should be the target of KAIZEN activities. The top of Fig. 1, Activities, shows that functions of Information Communication Technology (ICT) tools should be used for KAIZEN activities based on Plan, Do, Check, Action (P - D - C - A) cycles of Total Quality Management (TQM). Acquiring information from classes of various functions in the reference architecture of a manufacturing system is necessary for lean production systems, as shown in the bottom of Fig. 1. Especially, an information-interoperable environment is required that can acquire information from heterogeneous machinery at the device-level, and which relates to MES-level information. Moreover, use of information from the information-interoperable environment is required along with an easy and flexible programming environment of application systems depending on purposes of KAIZEN activities of lean production systems. 


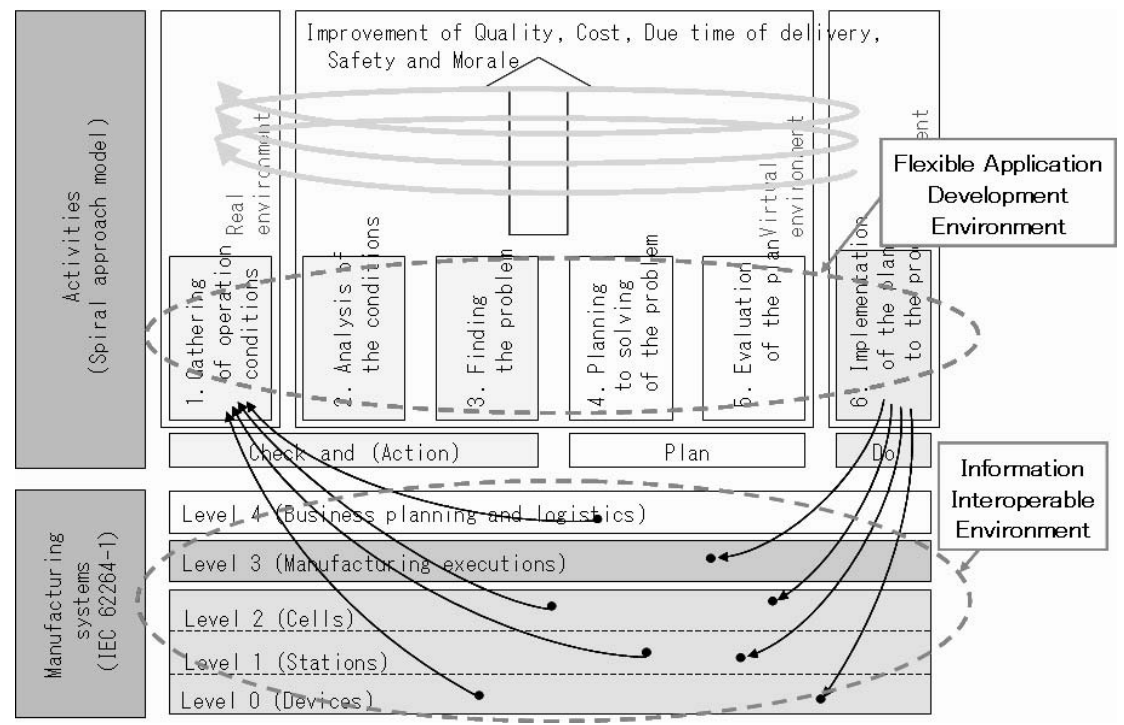

Fig. 1. Requirements of information-interoperable environment

\subsection{IIE for factories}

Two standard interfaces are provided by ORiN: an application interface for application system programs and a provider interface for resources such as devices and databases. In its kernel, ORiN has a device model called Controller Access Object (CAO). The IIE was developed using CaoSQL, which is middleware for ORiN's application systems to manage access control of information from devices and to record a history of variables of devices that are connected with the CAO.

The CaoSQL has a CaoSQLController class for discriminating among different devices; it also has a CaoSQLItem class for defining various types of information from devices. Information corresponding to CAO that is connected to multi-vendor machine tools, robots and Programmable Logic Controllers (PLCs) can be selected for KAIZEN activities of lean production systems and can be defined in CaoSQLItem objects and CaoSQLController objects. Moreover, MES-level information is definable in CaoSQLItem objects in connection with device-level information, as shown in Fig. 2.

Using the IIE with a web server, remote monitoring of device-level information and MES-level information through web applications can be customized easily by the user. Along with accumulation of data available from the device-level and MESlevel database histories, other application systems such as an operation management system can be created using a computer spreadsheet program (e.g., Excel; Microsoft Corp.), thereby granting users even greater freedom in their computing environments. Users can entrust IIE with information gathered from the device-level and MES-level. Software development costs can be reduced because users can thereby concentrate on development of application systems. 


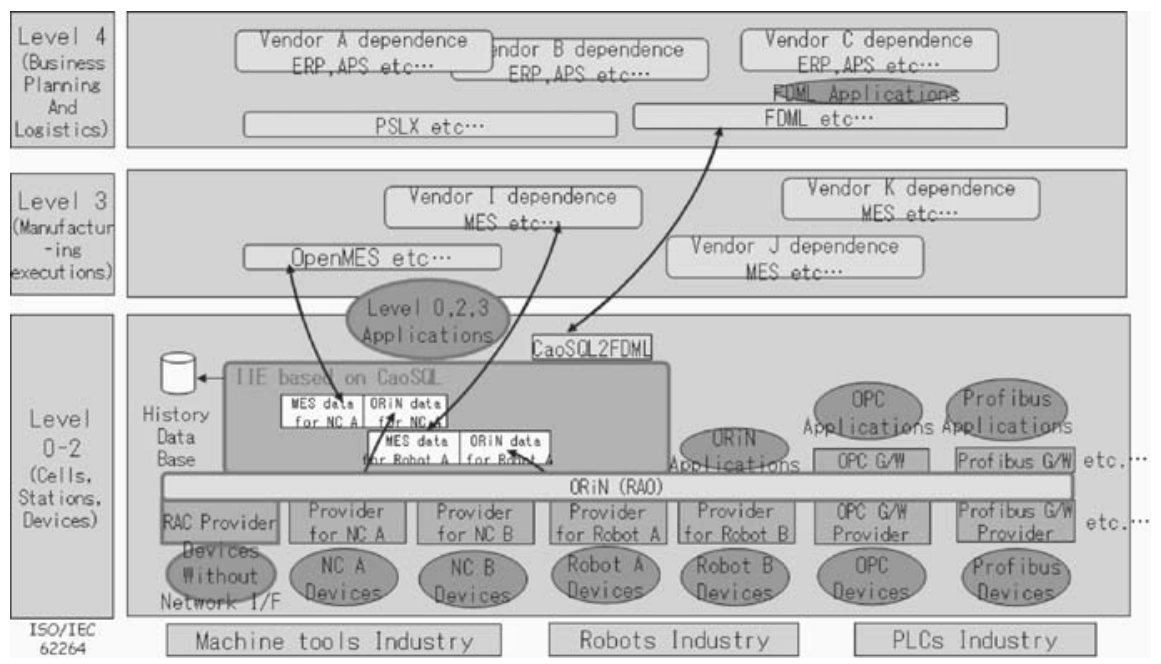

Fig. 2. Information-interoperable environment

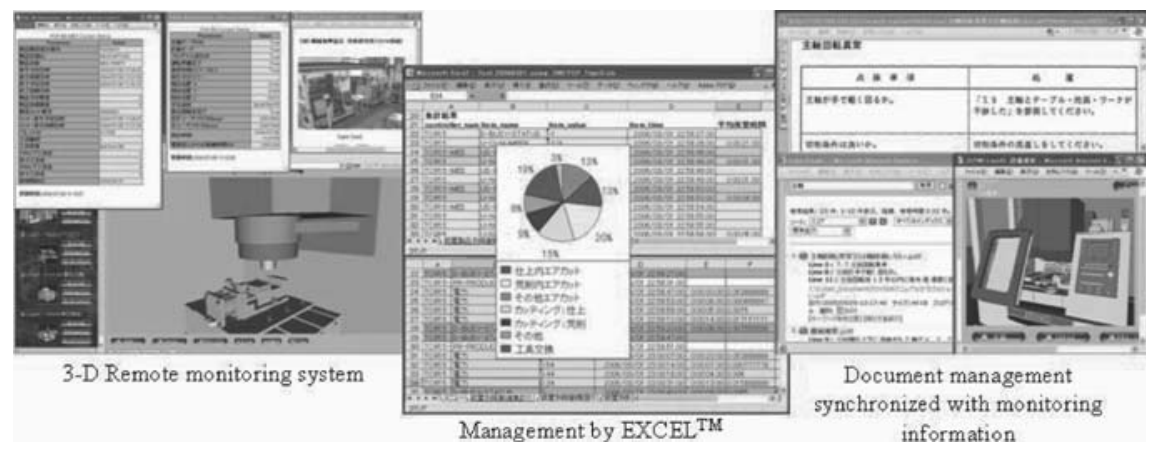

Fig. 3. Examples of application systems

Figure 3 depicts examples of application systems using the IIE. The left side of Fig. 3 shows a three-dimensional (3-D) remote monitoring system that can indicate the status of a manufacturing system through multimedia consisting of 3-D models, text, and still images together with MES-level information. With the accumulation of data available from the device-level and MES-level database histories, other application systems, such as an operation management system, can be created using a computer spreadsheet program (e.g., Excel; Microsoft Corp.), giving users even greater freedom in their computing environments, as shown in the middle panel of Fig. 3. The right panel of Fig. 3 shows a document management system that works with the 3-D remote monitoring system. 


\subsection{IIE for Inter-enterprise collaboration environment}

For inter-enterprise collaboration and for changing the IIE's information to FDML information, CaoSQL2FDML was developed. It can also send FDML information to the Factory Data Center (FDC) as a commercial Application Service Provider (ASP) service. Using the FDC, secure information and application-sharing services can be provided for inter-enterprise collaboration. This section provides an overview of FDML, and FDC.

The FDML is a de facto standard for describing information from the point of production. The FDML was proposed by the Manufacturing Science and Technology Center (MSTC) in Japan. Moreover, the FDML is based on eXtensible Markup Language (XML) technologies, which can facilitate description of information about a wide range of information such as device-level information, MES-level information, and business planning information. The FDML can manage information from devices that are dispersed in the factory by adding a time stamp to the information. Furthermore, FDML is widely and flexibly applicable to users' application systems through addition of logical information such as data names, data types, and maximum/minimum values to physical information. Table 1 shows that FDML has tags of XML such as InfoTag for describing sources of information, and DefinitionTag for describing the relation between physical information and logical information.

The FDC is an internet server that is used through access with FDML; it has some functions such as those of a hosting server, database, ASP, and a security function by Virtual Private Network (VPN). In addition, FDC is used for remote maintenance for multi-vendor manufacturing systems through inter-enterprise collaboration of device vendors and inter-enterprise collaboration in obtaining joint orders through access from multi-point users.

Table 1. FDML Tags

\begin{tabular}{|c|c|}
\hline Content of FDML & Description \\
\hline$<$ ?xml version=" 1.0" encoding=" Shift_JIS" $>$ & XML Declaration \\
\hline$<$ FDML version=" $1.02 ">$ & FDML start tag \\
\hline$<$ Info $></$ Info $>$ & Field machine information \\
\hline$<$ Definition $></$ Definition $>$ & $\begin{array}{l}\text { Correlation with physical channels } \\
\text { and logical channels }\end{array}$ \\
\hline$<$ Condition $></$ Condition $>$ & Condition to send FDML \\
\hline$<$ Data $></$ Data $>$ & Time-series field data \\
\hline$</$ FDML $>$ & FDML end tag \\
\hline
\end{tabular}

\subsection{Integration of both IIE for factories and Inter-enterprise collaboration}

Finally, CaoSQL2FDML was developed. It is a method for connection of the IIE and the FDC as one inter-enterprise collaboration environment. No information of whole IIE for factories is necessary with inter-enterprise collaboration. Therefore, 
information from the CaoSQLController class and CaoSQLItem class that is selected for inter-enterprise collaboration can be converted to FDML format and can be sent to FDC using CaoSQL2FDML. Figure 4 shows a conversion method of CaoSQL2FDML. Using this method, data names, data types, and maximum/minimum values of the CaoSQLController class and CaoSQLItem class of CaoSQL of the IIE are mapped to the Definition class of the FDML. In addition, values of the CaoSQLItem class are mapped to the Data class of the FDML.

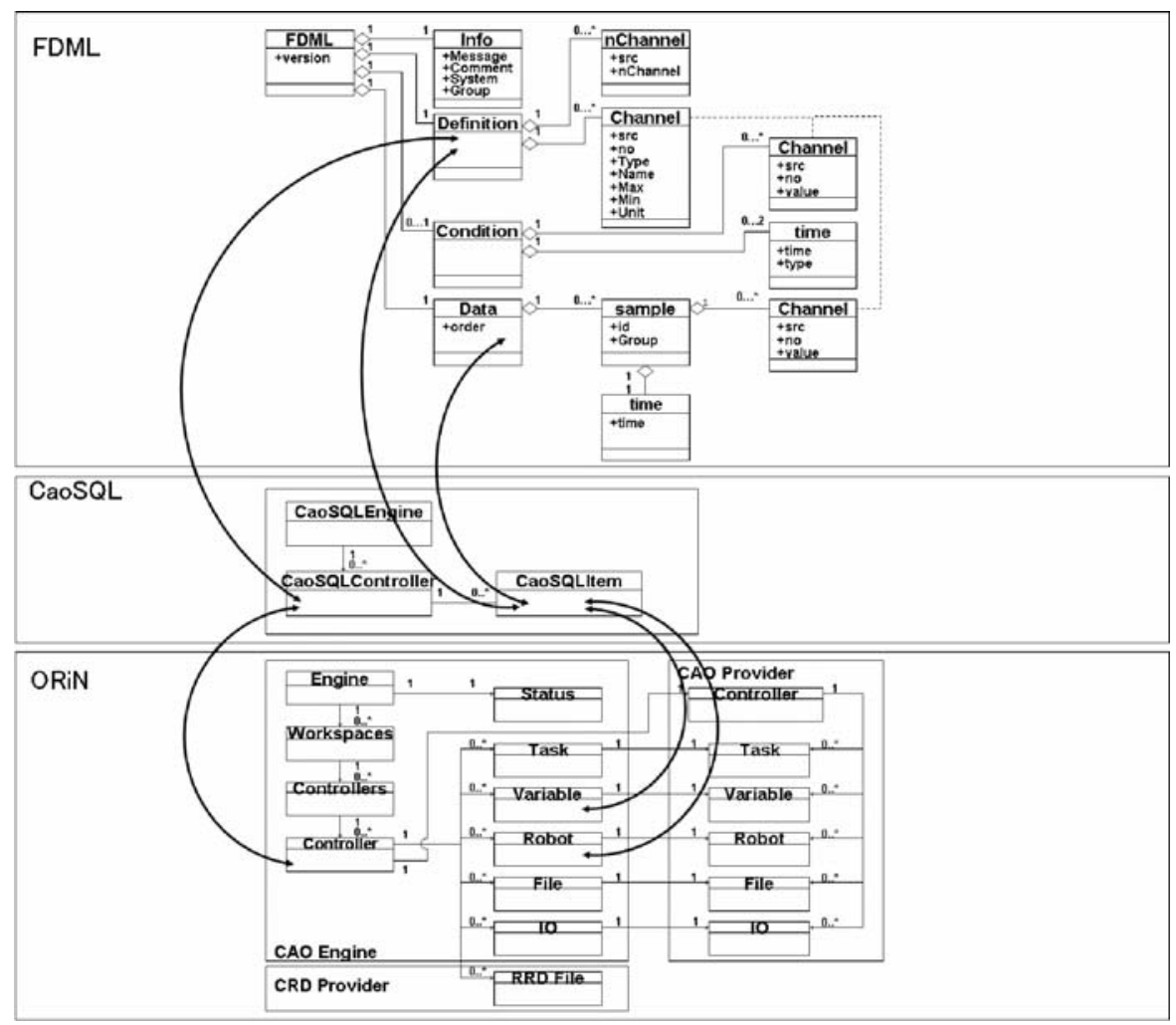

Fig. 4. Class chart mapping between CaoSQL and FDML

\section{Development of a prototype system based on the IIE}

A prototype system was developed based on the IIE and the CaoSQL2FDML. Evaluations of the prototype system were performed. Targets of the prototype system are a turning center and a multi-vendor manufacturing system line consisting of one transfer robot and two machine tools at Japan Society for the Promotion of Machine Industry (JSPMI, Higashikurume-city, Tokyo). The prototype system is integrated with the FDC server at NTT Corp. (Musashino-city, Tokyo), with a monitoring client at Tokyo Big Sight Inc. (Koto ward, Tokyo) using the internet, as shown in 
Fig. 5. Evaluations of remote monitoring from the client to the IIE were done directly. Evaluations of remote monitoring from the client to the FDC were also done using CaoSQL2FDML, considering inter-enterprise collaboration. Moreover, identity was evaluated using these monitoring results.

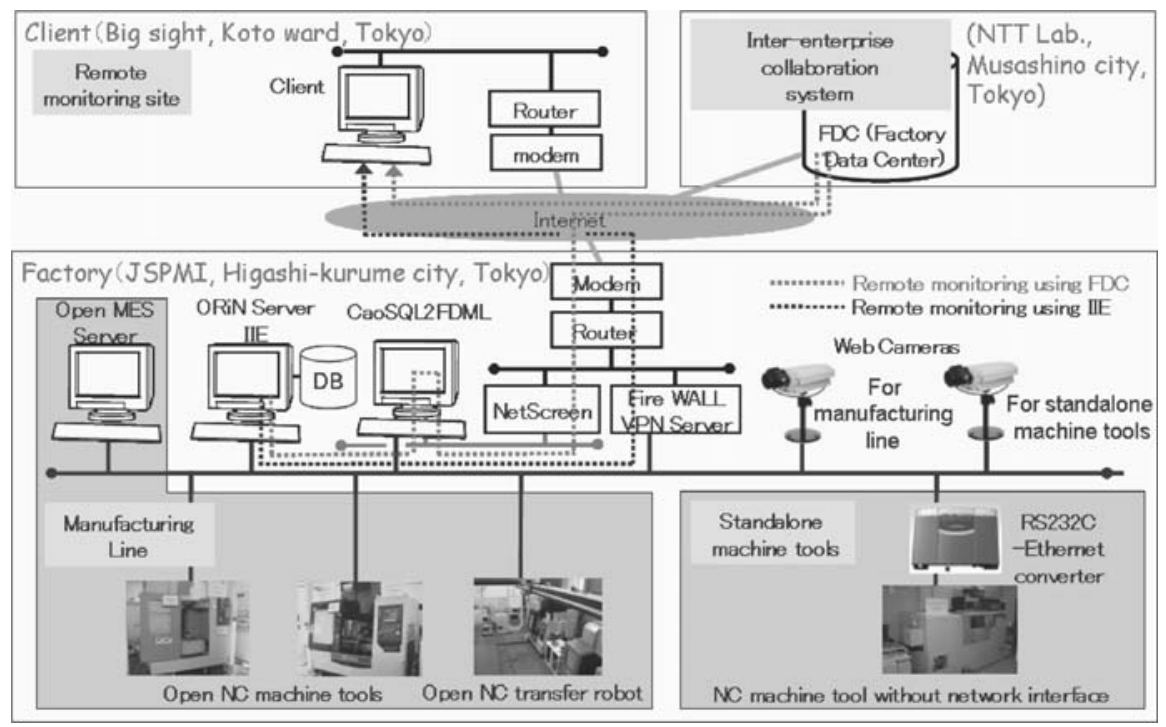

Fig. 5. Configuration of the prototype system

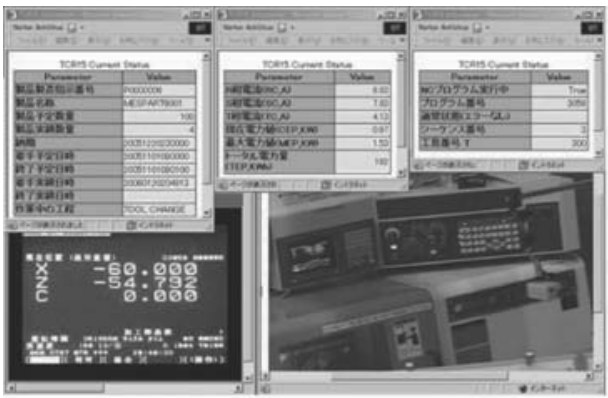

Monitoring using the IIE directly

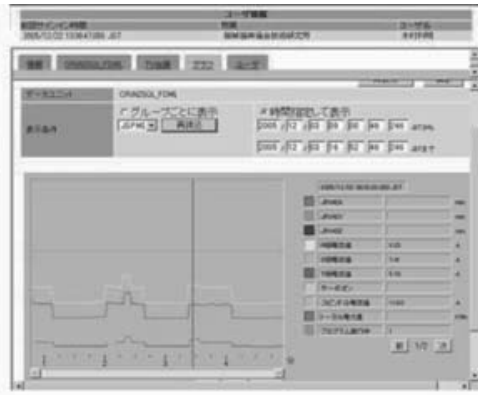

Monitoring using the FDC

Fig. 6. Test operation of the environment

The prototype system was exhibited at the 2005 International Robot Exhibition held in November 2005 at Tokyo Big Sight Inc. The client computer was able to stably monitor the prototype system at the JSPMI during the entire exhibition ( 4 days, $7 \mathrm{~h}$ /day), both using FDC and not using FDC. Moreover, the similarity of information using FDC and information not using FDC was confirmed. Figure 6 shows an example of the indicator screen for monitoring electrical consumption of the turning center at JSPMI on the client in the test operation. Results of this test 
operation confirmed that interconnection between the IIE and inter-enterprise collaboration system using FDML and FDC was possible and that the system can be used stably.

Moreover, it was confirmed that modification of the application system was easy. For example, in the case of application software of monitoring, target machines and variables for monitoring were altered by re-writing the name of the CaoSQLController object and/or CaoSQLItem object written in Script language in HTML file.

\section{Conclusion}

The IIE, which can connect device-level information and MES-level information for KAIZEN activates of the lean production system was developed using ORiN. It was confirmed that customizing the application system using the IIE was easy. Then, it was confirmed that IIE was suitable for lean production systems that require a flexible application development environment. Moreover, CaoSQL2FDML was developed for inter-enterprises collaboration, for changing the IIE's information to FDML information for use at an ASP. Moreover, application systems using the CaoSQL2FDML were developed. Results of this test operation confirmed that interconnection between the IIE and the inter-enterprise collaboration system using FDML and FDC is possible and can be used stably.

Several research results from IIE have been commercialized. These products are being used at customers' factories. In the future, not only application systems using IIE for factories but also application systems of inter-enterprise collaboration using the CaoSQL2FDML must be evaluated in actual factories.

\section{References}

1. ORiN: http://www.orin.jp/ (last accessed July 2006).

2. OPC: http://www.opcjapan.org/ (last accessed July 2006).

3. CC-Link: http://www.cc-link.org/eng/t_html/top.html (last accessed July 2006).

4. Profibus: http://www.profibus.com/ (last accessed July 2006).

5. DeviceNet: http://www.odva.org/ (last accessed July 2006).

6. T. Kimura and Y. Kanda, Development of an information-interoperable environment between device-level information and MES-level information, Proc. of APMS2005 (Advances in Production Management Systems), (2005).

7. H. Tezuka, Y. Nanjo, N. Katafuchi, Y. Nakamura, S. Iwaki, T. Machino, and K. Shimokura, Sensor and Robot Collaboration based on a Network Robot Platform, Proc. of Workshop on Network Robot System at International Conference on Robotics and Automation, (2005). 